\title{
Subthreshold Corazol Doses Induced Generalized Seizures in Audigenic Seizure-Prone Rats
}

\author{
Irina B. Fedotova, Natalia M. Surina, Georgy M. Nikolaev, Inga I. Poletaeva*
}

Biology Department, Lomonossov Moscow State Universi8ty, 119992, Moscow

*Corresponding author: Poletaeva, I.I. Biology Department, Lomonossov Moscow State Universi8ty, 119992, Moscow, E-mail: ingapoletaeva@mail.ru

\begin{abstract}
Purpose: It was suggested that high audiogenic epilepsy (AE) susceptibility in rats of Krushinsky-Molodkina (KM) rat strain could be accompanied by increase in general seizure proneness. This could be tested exploring the reaction to low corazol doses in these rats and in rats of genetically related but non-prone to AE strain (strain " 0 "), which was used as control,.

Method: 6 months old male rats of two strains (KM and "0") received the subthreshold $(40 \mathrm{mg} / \mathrm{kg}$ ) corazol injections. After injections rats were placed in individual cages and were observed for 40 - $50 \mathrm{~min}$ in order to discover the display/absence of corazol induced convulsions.

Results: The typical corazol seizures developed in more than $50 \%$ of KM rats (AE-susceptible) in comparison to non-prone control rats, in which these seizures were more rare events. The control rats of " 0 " strain demonstrated seizures of significantly lower intensity, than in KM rats. Although most rats of "0", which did not express corazol seizures and no AE seizures three weeks before the experiment as well, demonstrated they demonstrated the intense AE seizures when tested 20 min after corazol injection.

Conclusions: Rats of KM strain, selected for intense AE seizures, developed generalized seizures in response to subthreshold corazol dose, demonstrating the elevated general seizure proneness
\end{abstract}

Received Date: May 31, 2016

Accepted Date: July 04, 2016

Published Date: July 08, 2016

Citation: Poletaeva, I.I., et al. Subthreshold Corazol Doses Induced Generalized Seizures in Audigenic Seizure-Prone Rats. (2016) Int J Neurol Brain Disord 3(2): 49- 54.

DOI: $10.15436 / 2377-1348.16 .954$

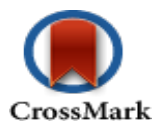

Keywords: Audiogenic epilepsy; Krushinsky-Molodkina rat strain; Strain “0”; Corazol; Seizure proneness

\section{Introduction}

Corazol (pentylenetetrazole) is the convulsant, which is used in experimental epilepsy studies in order to induce clonic and tonic seizures. The single small dose $\left(40 \mathrm{mg} / \mathrm{kg}\right.$ ) of corazol does not elicit seizures in rats (or mice) ${ }^{[1-3]}$. The higher doses of this drug (starting from $60 \mathrm{mg} / \mathrm{kg}$ ) induce the intense seizures in rats and mice with clonic and tonic components ${ }^{[1]}$.

The seizures induced by loud sound were described in rodents of different species and genotypes (audiogenic epilepsy, AE). These convulsions are especially intense and stable in "shape" in rodent strains selected for this trait ${ }^{[4]}$. Are the predispositions to these refractory seizures accompanied by general increase in seizure susceptibility? There are literature data which are in concordance with this idea - in audiogenic seizure prone rats (Krushinsky-Molodkina -KM) the hippocampal electric seizure thresholds correlated significantly with AE intensity ${ }^{[5]}$ and the similar correlation was described for Wistar Audiogenic Rats - WAR ${ }^{[6]}$. At the same time the AE morphological substrate, rather well investigated ${ }^{[7]}$, and genetic factors, which underlie this trait, are presumably not fully identical to those for general seizure syndrome which could be observed as the response to chemicals or electroshock ${ }^{[8-11]}$.

The goal of the present study was to compare the AE proneness and corazol-induced seizures, induced by low doses of this convulsant, in rats of two strains, which differed by the AE proneness. 


\section{Material and Methods}

\subsection{Experimental animals}

The 4 - 6 months old male rats of Krushinsky-Molodkina (KM) strain were used $(n=31)$, as well as age matched males of strain " 0 " $(\mathrm{n}=33)$ in 4 experimental series. Rats were maintained in plastic T4 cages $(545 \times 395 \times 200 \mathrm{~mm}$ for $1 \times$ b x h), $5-7$ animals in each with natural day-night cycle and tap water and food (Laborator-korm and MEST, Moscow, Russia) ad lib. All experimental manipulations were performed at the same 24-hour time period - from 15 to 17 p.m. KM strain. The KM AE-prone strain (now demonstrating the highest expressivity and penetrance characteristics) was derived from Wistar stock in late 1940-s. The AE-prone KM strain is the inbred strain which is maintained by brother-sister mating schedule. Animals from F69 and F70 were used in present experiments.

AE-non prone strain " 0 ". The strain " 0 " was selected recently ${ }^{[12]}$ for the lack of AE seizures, using the F2 population of KM x Wistar cross (with 2 backcrosses on KM parental strain at the initial selection generations) as the basal one. These rats are also maintained by brother-sister mating schedule for several generations although the inbred status of this strain had not been yet confirmed. Animals from F29 and F30 selection generations were used in this study.

The four experimental series were the following: The series 1 was performed using KM rats from F69 inbred generation and strain "0" animals from F29 selection generation. Series 2 followed in 2 months (using another cohort of KM rats of the same generation), while series 3 and 4 were performed 6 months later (in winter), using the animals of the next generations.

Series 1: The $40 \mathrm{mg} / \mathrm{kg}$ corazol dose was injected to KM strain rats $(\mathrm{n}=7)$ and to" 0 " strain rats $(\mathrm{n}=8)$. After the injection animals were placed into the individual cages and their behavior (plausible intense allogrooming, myoclonic jerks and clonic-tonic convulsions) was observed with manual recording the seizure latency and seizure intensity. Animals were observed either for 60 min (rats of KM strain), or tested for AE in 20 min after injection (rats of " 0 " strain).

Series 2: Corazol $(40 \mathrm{mg} / \mathrm{kg})$ was injected to rats of $\mathrm{KM}$ strain $(\mathrm{n}=10)$, which were under observation up to seizure start or as described above.

Series 3: Corazol $(40 \mathrm{mg} / \mathrm{kg})$ was injected to rats of KM strain $(\mathrm{n}=14)$ and to rats of strain" 0 " $(\mathrm{n}=11)$. They were under observation up to seizure start or as described above.

Series 4: Rats of strain ' 0 ' $(\mathrm{n}=14)$ were corazol injected $(80 \mathrm{mg} / \mathrm{kg})$ and were under observation up to seizure start or as described above.

During all series two experimenters performed the evaluation of seizure occurrence and intensity (see below) in experimental animals. Their independent scores, based on visual observations, were compared and the coincidence of two evaluations was found in all cases. The experiments were performed according to rules of EC 2010 Directive, and they were approved by Bioethic Counsel of Lomonossov Moscow State University (January 14, 2016).

\subsection{Drug injections}

Corazol (pentylenetetrazole)(Sigma-Aldrich) $40 \mathrm{mg} / \mathrm{kg}$ in saline was injected i. p. In series 4 the rats of " 0 " strains were given the higher (convulsive) dose $-80 \mathrm{mg} / \mathrm{kg}$. Each rat was placed into individual cage immediately after the injection (see above). The arbitrary scale, used to measure the corazol seizure intensity was different from that introduced by Velisek et al. ${ }^{[13]}$ earlier, as the low dose of corazol used did not permit to notice the development of seizures in details, which is required by Velisek et al. ${ }^{[13]}$ scale. The scale used in this study implied " 1 " as solitary myoclonic jerks, " 2 " - the intense myoclonic and generalized clonic seizures of the trunk, and " 3 " - the tonic seizures of all muscles.

\subsection{AE testing}

The sound exposure (120 dB sound of the "auditory bell") was performed in sound attenuating plastic box (40 x 30 x 50 $\mathrm{cm})$. The standard robust "auditory" bell was used during all years of KM strain selection although the control measurement of sound intensity was performed at the begin of 2015. Microphone B \& K 4135 together with acoustic pressure measurement (B \& K 2235, Brüel \& Kjær) were used to determine the sound intensity. No special measurement of sound intensity before each sound exposure was performed. The sound was switched on up to the development of seizure of maximal intensity, or, in case when no seizures appeared, up to $90 \mathrm{~s}$. The latencies of consecutive seizure stages AE as well as maximal seizure intensity and seizure duration were recorded manually. AE intensity was evaluated according to arbitrary scale adopted for long time in the Laboratory of physiology and genetics of behavior: " 0 " -lack of reaction during $90 \mathrm{~s}$, " 1 " - wild run stage, " 2 " - clonic seizures, developed on the belly, " 3 " clonic-tonic seizures as the animal fall on the side, " 4 " - tonic seizures of the whole body muscles with short breath arrest ${ }^{[4]}$. The preliminary testing of rats of both strains (performed 1.5 months before the experiment) demonstrated that KM rats developed AE seizures of highest intensity while " 0 "rats tested 3 times (with 5 - 7 days intervals) demonstrated no seizures in response to sound.

\subsection{Statistics}

The nonparametric Mann-Whitney criterion was used in order to compare the corazol seizure intensity in two strains (Statistica-6), $\mathrm{p}<0.05$ was taken as the significant difference. 


\section{Results}

\subsection{AE proneness}

As mentioned in the previous section, all $\mathrm{KM}$ rats used in the experiments demonstrated the maximal AE intensity (tonic seizures), while all rats of strain "0" demonstrated "0" reaction to sound - no AE.

\subsection{The data obtained in this study for KM and "0" rat strains}

Animals reactions to subconvulsive corazol dose $(40 \mathrm{mg} / \mathrm{kg})$, are summarized in table 1 . The details of these experiments (series 1 - 4) are presented below (3.3).

Table 1: The seizure incidences in rats of KM and "0" strains in response to subconvulsive dose of corazol (i.p. injections, n- number of animals)

\begin{tabular}{|l|l|l|l|}
\hline Seizure Indices & KM $(\mathbf{n}=\mathbf{3 1})$ & $\mathbf{0}(\mathbf{n}=\mathbf{1 9})$ & Statistical significance (Mann-Witney criterion) \\
\hline Seizure intensity (arbitrary units)* & $2,35 \pm 0,22$ & $0,58 \pm 0,16$ & $\mathrm{p}<0.001$ \\
\hline Animals with seizures (n and \%) & $90,3 \%(\mathrm{n}=28)$ & $52,6 \%(\mathrm{n}=10)$ & $\mathrm{p}<0.01$ \\
\hline Seizure latency (s) & $221,07 \pm 17,9$ & $280 \pm 81,2$ & n.sign \\
\hline
\end{tabular}

*The seizure intensity arbitrary scale used in this study includes the following degrees: 1 - single myoclonic jerks, 2 - intense myoclonus replaced by clonic seizures, 3 - the tonic seizures of all trunk and extremities' muscles (and "0" for lack of seizures) (see Material and Methods).

Series 1: The corazol dose $40 \mathrm{mg} / \mathrm{kg}$ induced the intense "corazol" tonic seizures in 6 out of 7 rats of KM strain. The seizures developed within 14 min after the injection with different latencies.

Preliminary observations (data not shown) demonstrated that in case of $40 \mathrm{mg} / \mathrm{kg}$ rats of " 0 " strain develop no seizure being watched during $60 \mathrm{~min}$. As it was crucial to "catch" some changes in the brain excitability which could promote audiogenic seizures rats of "0" strain in present experiments were sound exposed after 20 min after the PTZ injection. In all 8 rats of strain "0"the corazol injection did not provoke any signs of seizures. After 20 min elapsed these animals were exposed to loud sound in order to test their AE. 4 out of 8 animals displayed the typical AE seizures with minimal latency $(1-2 \mathrm{~s})$ and the highest intensity - tonic seizure of trunk and extremities. It should also be reminded in all these animals no audiogenic seizures were not registered after triple sound exposures with intervals of $5-7$ days. The wild run seizure stage was replaced by tonic seizure stage very quickly ( $7-22$ s). In 3 cases out of 4 the tonic AE seizures were of the unusual pattern - they developed as the animal lied on the back, the phenomenon, which was never seen before.

Series 2: 10 rats of KM strain were injected with corazol $(40 \mathrm{mg} / \mathrm{kg}$ ). All animals displayed corazol seizures (within $10 \mathrm{~min}$ after injection), and in 7 cases these seizures were of maximal intensity (score 3 - tonic seizures of trunk and extremities).

Series 3: Corazol $(40 \mathrm{mg} / \mathrm{kg})$ was injected to rats of both strains and practically all animals demonstrated the reaction to corazol injections. In 11 (out of 14) rats of KM strain the intensity of corazol seizures was maximal (arbitrary score- 3), with mean latency $201 \pm 31.7$ s. In strain " 0 " rats the seizures of low intensity (myoclonic jerks and short clonic seizures) were noted in 9 animals (out of 11) with longer latency (see table 1). One rat of "0" strain demonstrated the only one myoclonic jerk, in 6 rats the series of myoclonic jerks was noted, while in 2 animals the clonic seizure of short duration developed (with $300 \mathrm{~s}$ and $900 \mathrm{~s}$ latencies respectively).Being expressed in arbitrary units, the corazol seizures in rats of strain "0" were significantly less intense, in comparison to KM rats (see table 1).

In this experimental series three KM rats did not develop seizures in response to sub-convulsive corazol dose. In 20 min after the injection they were exposed to sound stimulation (see Methods, 2.3). In these animals the AE tonic seizure stage developed with 5 - 7 s latencies without displaying the typical AE wild run stage. In 2 rats of strain ' 0 ' which did not develop reaction to corazol injection the sound exposure did not elicit the AE seizure at all.

Series 4: The reaction of strain " 0 " rats $(\mathrm{n}=14)$ to corazol convulsive dose $(80 \mathrm{mg} / \mathrm{kg})$ was analyzed. In 2 animals no corazol seizures were noted, 2 rats displayed clonic seizures (arbitrary score 2) while the rest 10 animals demonstrated the intense tonic corazol seizures. The mean latency of seizure development (in affected animals) was $390 \pm 162.8 \mathrm{~s}$ (with maximum $-2040 \mathrm{~s}$ and minimum- $60 \mathrm{~s}$ ).

\section{Discussion}

Several aspects of experimental data presented need to be discussed. In general corazol treatment revealed the increased seizure susceptibility in AE prone rats. These effects were demonstrated both in WAR strain, which was bred from Wistar population $^{[6]}$, and in GEPR (derived from Sprague-Dawley rats) ${ }^{[14]}$. In these studies the increased proneness to pilocarpine-induced and to electroconvulsive seizures was clearly shown. No detailed analysis of the elevated general seizure susceptibility was performed, although the "forebrain" localization of corazol effects was demonstrated explicitly ${ }^{[15]}$. At the same time it was demonstrated that in 
rat pups with previous (24 hours or 20 days earlier) history of the febrile seizures, corazol ( $80 \mathrm{mg} / \mathrm{kg}$ ) developed less intense seizures than in controls, demonstrating the "therapeutic" effect of neonatal hyperthermia ${ }^{[16]}$, presumably by inducing changes in heat-shock proteins expression in brain ${ }^{[17]}$.

The main result of the study presented is that rats of KM strain (highly AE-prone) were sensitive to subconvulsive corazol dose and demonstrated seizures in majority of cases (28 animals with seizures versus 3 without it). Rats of strain " 0 ", tested for AE expression in preliminary experiments, demonstrated no AE seizures (being tested three times successively). Although in the $1^{\text {st }}$ series 4 animals of " 0 " strain (out of 8) displayed the "full” AE seizure (after subconvulsive corazol!). In series 3 " 0 " rats demonstrated corazol seizures of much lower intensity than KM rats (table 1). Their reaction to convulsive $(80 \mathrm{mg} / \mathrm{kg}) \mathrm{corazol} \mathrm{dose}$ was also found. Thus the prevalence of elevated general seizure susceptibility of AE-prone rats (KM strain) over rats, which initially demonstrated no AE seizures, is evident.

Although the data evidence the elevated seizure susceptibility to low dose of convulsant in " 0 " rats as well. The probable explanation of this fact is that these animals shared the KM genetic background in larger proportion than with Wistar rats. At the initial selection generations two backcrosses of KM-Wistar hybrid offspring to KM rat parental strains were performed ${ }^{[12]}$. These back crosses were undertaken in order to increase the genetic background similarity between KM and " 0 " strains. This goal was more or less attained and it was demonstrated, that differences in AE-proneness between KM and " 0 " were not accompanied by differences in anxiety and depression indices in the respective tests ${ }^{[18]}$. The genetic relatedness of " 0 " strain to KM genotype had been manifested in the data of present study - rats of " 0 " strain manifested seizures of low intensity in response to subconvulsive corazol injection in about $50 \%$ of cases.

The differences between series 1 and 3 in the proportions of animals which developed seizures (in " 0 " rats, in particular) could be due to the fact, that first two series were performed during summer season, in contrast to winter time when series 3 took place. The selection success for strain " 0 " was not linear in the course of generations ${ }^{[12]}$ and the increase in seizure proneness in this strain could be observed (probably as the seasonal variability as well).

Corazol (pentylenetetrazole) is the antagonist of GABA-A ionotropic receptors, as it inhibits GABA-dependent chlorine channels in dose- and potential-dependent way (Macdonald, Barker, 1978). Corazol binds to GABA-A receptor benzodiazepine site (Walsh et al, 1999) ${ }^{[18-21]}$.

Although the data were obtained as well which demonstrated the influence of this drug on metabotropic GABA-B receptor $^{[22]}$. It is the common knowledge that $\mathrm{AE}$ is determined (at least partly) by the excitatory and inhibitory neurotransmitter misbalance, and the participation of brain GABAergic system being very important in particular (Faingold et al., 1994) ${ }^{[7,23-26]}$. This could determine the increased vulnerability to corazol seizures of KM rats as highly AE prone animals.

The data of the present study confirmed that AE proneness in KM rats is associated with high seizure corazol seizure propensity. Corazol injections usually provoke the development of seizure activity in limbic brain structures (e.g. Galvis-Alonso et al., $2004)^{[27]}$. Although in reality (as the data presented show) the pattern of corazol action is more complicated. Our experiments demonstrated that rats of strain " 0 " (non-prone to AE) started to react by seizures in response to loud sound after sub-convulsive corazol dose. This means that the increase in seizure susceptibility after corazol treatment could affect not only limbic brain structures (presumably responsible for myoclonic and clonic seizures), but also brain stem substrate of AE tonic seizures. AE seizures take start in acoustic brain stem with further irradiation to inferior colliculi and motor brain stem and basal ganglia substrates. Previously the significant interstrain (KM vs "0"rats) differences were found in the striatal density of D2- and NMDA -receptors ${ }^{[28]}$. The higher density of these receptors in KM rats could also make the impact in the increased proneness of KM rats to seizures of other nature (i.e. reaction to chemical convulsant). These differences could also be responsible for the abnormal development of AE pattern in 3 cases out of 14, series 3, when the wild run stage of AE was "omitted" in KM rats with no corazol seizures. The ultra-rapid expression of AE seizures in these cases could be one more indication that the corazol effects are connected to dopamine brain function, as the indirect indications of this phenomenon were reported by other authors ${ }^{[2-32]}$.

The low corazol dosages induced in " 0 " rats the non-specific increased seizure proneness as these rats start to respond to loud sound stimulation by AE seizure (being insensitive to this treatment in the "background" state). Such development of audiogenic seizures in rats of this strain could be due (as mentioned before) to their genetic origin. The " 0 " rats were derived from the F2 hybrid population (KM x Wistar) by means of selection for the trait "lack of AE"[12]. The proportion of strain " 0 " rats with no AE seizures in response to sound is still not high (at the level of selection generation tested) and was not higher that $40 \%$ in each generation (the rats, chosen for the experiments were from the non-prone proportion of these animals). This means that the trait (lack of AE) is of not high penetrance. It is also important that AE seizures in " 0 " strain (approximately in $60 \%$ of animals in each generation) are not intense, usually they consist of wild run and clonic seizures, but not of tonic seizures. Although in the experiments presented the really " 0 " rats demonstrated the prompt intense AE tonic seizures after sub-convulsive corazol dose.

The sub-threshold doses of convulsant (corazol) are usually applied according to chronic schedule in order to induce the gradual increase of seizure proneness (kindling). Although in our experiments this single dose was shown to be capable to induce the full developed corazol-seizure pattern in animals with increased AE proneness.

\section{Conclusion}

The interstrain differences, revealed in this study, indicate the high unspecific seizure proneness of KM strain rats. This also could mean that AE seizures, from one side, and convulsions in response to drug-induced GABA receptors inactivation, from another, have probably the common links in their mechanisms. This, in turn, could signify, that rodent AE represents the valuable and important model for the investigation of epileptogenesis. 
The study was supported by RFBR (grant \# 04-15-01732 and State program \# AAAA-A16-116021660055-1

\section{Highlights}

Audiogenic-prone rats (KM) develop seizure after subconvulsive corazol injection

In non-audiogenic rats ("0"), corazol induced non-intense reaction

After subconvulsive corazol dose "0" rats developed intense audiogenic seizures

\section{References}

1. Coppola, G., Arcieri, S., D'Aniello, A., et al. Levetiracetam in submaximal subcutaneous pentylentetrazol-induced seizures in rats. (2010) Seizure 19(5): 296-299.

2. Kumar, A., Lalitha, S., Mishra, J. Possible nitric oxide mechanism in the protective effect of hesperidin against pentylenetetrazole (PTZ)-induced kindling and associated cognitive dysfunction in mice. (2013) Epilepsy Behav 29(1): 103-111.

3. Zhao, R.R., Xu, X.C., Xu, F., et al. Metformin protects against seizures, learning and memory impairments and oxidative damage induced by pentylenetetrazole-induced kindling in mice. (2014) Biochem Biophys Res Commun. 448(4): 414-417.

4. Poletaeva, I.I., Surina, N.M., Kostina, Z.A., et al. The Krushinsky-Molodkina rat strain: The study of audiogenic epilepsy for 65years. (2015) Epilepsy Behav S1525-5050(15): 00251-00256.

5. Zorina, Z.A., Fless, D.A. Pharmacological analysis of the mechanism of myoclonic convulsions in rat. (1969) Bull Exper 67(1): 48-50.

6. Scarlatelli-Lima, A.V., Magalhães, L.H., Doretto, M.C., et al. Assessment of the seizure susceptibility of Wistar Audiogenic rat to electroshock, pentyleneterazole and pilocarpine. (2003) Brain Res 960(1-2):184-189.

7. Faingold, C.L. Neuronal networks in the genetically epilepsy-prone rat. (1999) Adv Neurol 79: 311-321.

8. Kupferberg, H. Animal models used in the screening of antiepileptic drugs. (2001) Epilepsia 42(Suppl 4): 7-12.

9. Löscher, W. Critical review of current animal models of seizures and epilepsy used in the discovery and development of new antiepileptic drugs. (2011) Seizure Jun 20(5): 359-368.

10. Maxson, S.C. A genetic context for the study of audiogenic seizures. (2016) Epilepsy Behav S1525-5050(15)00686: 1.

11. Serikawa, T., Mashimo, T., Kuramoro, T., et al. Advances on genetic rat models of epilepsy. (2015) Exp Anim 64(1):1-7.

12. Fedotova, I.B., Kostyna, Z.A., Surina, N.M. Laboratory rat selection for the trait "the absence of audiogenic seizure proneness". (2012) Russ J Genet 48(6): 685-691.

13. Velisek, L., Kubova, H., Pohl, M. Pentylenetetrazol-induced seizures in rats: an ontogenetic study. (1992) Naunyn-Schmiedeberg's Arch Pharmacol 346(5): 588-591.

14. Eells, J.B., Clough, R.W., Browning, R.A.,et al. Comparative fos immunoreactivity in the brain after forebrain, brainstem, or combined seizures induced by electroshock, pentylenetetrazol, focally induced and audiogenic seizures in rats. (2004) Neuroscience 123(1): 279-292.

15. Szyndler, J., Maciejak, P., Turzyn, D. Mapping of c-Fos expression in the rat brain during the evolutionof pentylenetetrazol-kindled seizures. (2009) Epilepsy Behavior16(2): 216-224.

16. Gonzalez-Ramirez, M., Salgado-Ceballos, H., Orozco-Suarez, S.A., et al. Hyperthermic seizures and hyperthermia in immature rats modify the subsequent pentylenetetrazole-induced seizures.(2009) Seizure 18 (7): 533-536.

17. Khudik, K.A., Pastukhov, Iu.F., Guzhova, I.V.,. Effects of thermal preconditioning on convulsive activity in rats with inheritable form of audiogenic epilepsy. (2011) Ross FiziolZhIm I M Sechenova 97(11):1237-1246.

18. Sarkissova, K.Yu., Fedotova, I.B., Surina, N.M., et al. Effect of chronic fluoxetine treatment on audiogenic epilepsy, symptoms of anxiety and depression in rats of four lines. (2016) Doklady Biological Sciences 467(1): 55-58.

19. Mimaki, T., Yabuuchi, H., Laird, H. et al. Effects of seizures and antiepileptic drugs on benzodiazepine receptors in rat brain. (1984) Pediatr Pharmacol 4(4): 205-211.

20. Vlainic, J., Pericic, D. Effects of acute and repeated zolpidem treatment on pentylenetetrazole-induced seizure threshold and on locomotor activity: Comparison with diazepam. (2009) Neuropharmacology 56(8): 1124-1130.

21. Ahmadiani, A., Mandgary, A., Sayyah, M. Anticonvulsant effect of flutamide on seizures induced by pentylenetetrazole: involvement of benzodiazepine receptors. (2003) Epilepsia 44(5): 629-635.

22. De Sarro, G., Palma, E., Costa, N., et al. Effects of compounds acting on GABA(B) receptors in the pentylenetetrazole kindling model of epilepsy in mice. (2000) Neuropharmacology 39(11): 2147-2161.

23. Kash, S.F., Johnson, R.S., Tecott, L.H., Noebels, J.L., et al. Epilepsy in mice deficient in the 65-kDa isoform of glutamic acid decarboxylase. (1997) Proc Natl Acad Sci 94(25):14060-14065.

24. Prieto-Martín, A.I., Aroca-Aguilar, J.D., Sánchez-Sánchez, F., et al. Molecular and neurochemical substrates of the audiogenic seizure strains: The GASH:Sal model. (2015) Epilepsy Behav S1525-5050(15)00276-0.

25. Rossetti, F., Rodrigues, M.C., de Oliveira, J.A., et al. Behavioral and EEG effects of GABAergic manipulation of the nigrotectal pathway in the Wistar audiogenic rat strain. (2011) Epilepsy Behav22(2): 191-199.

26. Semiokhina, A.F., Fedotova, I.B., Poletaeva, I.I. Rats of Krushinsky-Molodkina strain: studies of audiogenic epilepsy, vascular pathology, and behavior. (2006) Zh Vyssh Nerv Deiat Im I P Pavlova 56(3): 298-316.

27. Galvis-Alonso, O.Y., Cortes De Oliveira, J.A., Garcia-Cairasco N. Limbic epileptogenicity, cell loss and axonal reorganization induced by audiogenic and amygdala kindling in wistar audiogenic rats (WAR strain). (2004) Neuroscience 125(3): 787-802. 
28. Firstova, J.J., Abaimov, D.A., Surina, N.M., et al. Binding of specific ligand by D2- and NMDA-receptors of striatum cells in two rat strains predisposed and resistant to audiogenic seizures. (2012) Bull Exp Biol Med 154(2): 196-198.

29. Tchekalarova, J., Sotiriou, E., Angelatou, F. Down-regulation of dopamine D1 and D2 receptors in the basal ganglia of PTZ kindling model of epilepsy: effects of angiotensin IV. (2004) Brain Res1024(1-2): 159-166.

30. López-Grancha, M., Lopez-Crespo, G., Sanchez-Amate, M.C., et al. Individual differences in schedule-induced polydipsia and the role of gabaergic and dopaminergic systems. (1984) Psychopharmacology (Berl) 197(3): 487-498.

31. Diehl, R.G., Smialowski, A., Gotwo, T. Development and persistence of kindled seizures after repeated injections of pentylenetetrazol in rats and guinea pigs. (1984) Epilepsia 25(4): 506-510.

32. Tian, F.F., Zeng, C., Ma, Y.F., et al. Potential roles of Cdk5/p35 and tau protein in hippocampal mossy fiber sprouting in the PTZ kindling model. (2010) Clin Lab 56(3-4): 127-136.

Online ISSN: $2377-1348$

Journal Title: International Journal Neurology and Brain Disorders Journal Short Name: Int J Neurol Brain Disord
Ommega Online Publishers

E-mail: neurology@ommegaonline.com

Website: www.ommegaonline.org 